\title{
SURVEY ON VARIOUS PERSPECTIVES OF RAMAN AMPLIFIERS
}

Sumathy Raju

Assistant Professor, ECE, Kalasalingam Academy of Research and Education,

Virudhunagar, Tamil Nadu, (India).

E-mail: sumathyraju1110@gmail.com ORCID: https://orcid.org/0000-0001-9871-1780

Muthukumar Arunachalam

Associate Professor, ECE, Kalasalingam Academy of Research and Education,

Virudhunagar, Tamil Nadu, (India). E-mail: muthuece.eng@gmail.com ORCID: https://orcid.org/0000-0001-8070-3475

\section{Citación sugerida:}

Raju, S., y Arunachalam, M. (2020). Survey on various perspectives of raman amplifiers. 3C Tecnología. Glosas de innovación aplicadas a la pyme. Edición Especial, Marzo 2020, 247-259. http://doi. org/10.17993/3ctecno.2020.specialissue4.247-259

\section{Suggested citation:}

Raju, S., \& Arunachalam, M. (2020). Survey on various perspectives of raman amplifiers. 3C Tecnología. Glosas de innovación aplicadas a la pyme. Edición Especial, Marzo 2020, 247-259. http://doi. org/10.17993/3ctecno.2020.specialissue4.247-259 


\section{ABSTRACT}

Raman Amplifier (RA) is the fiber amplifier that follows Stimulated Raman Scattering (SRS) mechanism. For broadband amplification it is used, because of low noise and better gain. Raman amplification was investigated in multiple views. Many research works had focused in the views of pumping schemes, gain flattening, transmission system and noise analysis. In this paper, Raman amplification is studied in the views of varying Refractive Index profile of core, varying core gap radius, hybrid combination of RA with Erbium Doped Fiber Amplifier (EDFA). This paper also studies the investigations of this hybrid combination in Dispersion compensation at $\mathrm{C}$ and $\mathrm{S}$ bands, recycling of pump power and location of EDFA.

\section{KEYWORDS}

Refractive index profile, Hybrid Amplifiers, Dispersion compensation. 


\section{INTRODUCTION}

In long distance Optical communication system, whenever the signal strength becomes low, we need to boost up it. At the beginning, Regenerators were used to rejuvenate the signal. Here, the optical signal is converted into the electrical signal. After the regeneration, the electrical signal is again converted into optical signal which is a very costly process. Then Semiconductor Optical Amplifiers (SOA) were used. From the studies (Elndash, Mohammed \& Rashed 2010; Fugihara \& Pinto, 2008), these amplifiers have disadvantages like, low output power, high noise figure. In order to avoid these, Fiber amplifiers were used. Fiber amplifiers are the amplifiers where a fiber itself becomes amplification medium by using pumping. There are two fiber amplifiers are mostly used. One is EDFA and second one is Raman Amplifier. In EDFA, a rare earth element Erbium is doped. When EDFA is compared with RA, gain bandwidth is low and wavelength of operation is limited. But in RA, at any wavelength we can obtain amplification. So Raman amplifications are now mostly used in long distance optical communication system. The researches (Bromage, 2004; Islam, 2002; Namiki \& Emori, 2001) concluded that Raman amplification has important advantages of having very low noise and suitable for broadband applications, particularly in WDM systems, where simultaneous amplification is required for multi-channel light. Raman amplification may be Distributed Raman amplification (DRA) or lumped Raman amplification. Dhir and Gupta (2013), found that DRA has benefits like high gain, high data rate and less fiber loss. Raman amplifier also provides better performance when it is combined with EDFA or SOA. Many research works had been done in various aspects like flat gain amplification, noise performance, pumping schemes, \& hybrid system. Lot of ideas to pump powers and wavelengths selection was discussed in the literatures (Ferreira, Cani, Pontes \& Segatto 2011; Neto, Teixeira, Wada, \& André, 2007). Raman amplification is happening from the process SRS. SRS is a notable non-linear effect which affects the Signal to Noise Ratio (SNR) in a WDM system. It can also be used for amplification of the optical signals in a long distance optical communication link. The spontaneous Raman scattering was found by Sir C. V. Raman. In case of this scattering, a small quantity of the incident light is changed into light signal of either low or high frequency. SRS gives the amplification if the pump signal with suitable wavelength enters the fiber (Dhir et al., 2013; Ferreira et al., 2011; Fugihara et al., 2008). In SRS, photon form pumping source is 
absorbed by material and emits a photon with energy at its vibrational state. In fact, energy is transmitted from a high frequency optical signal to lower frequency optical signal (Dhir and Gupta, 2014). Raman amplification is not only possible for single mode fiber but also for multimode fiber which was discussed in a research (Polley \& Ralph, 2007). In this work, For fundamental mode LP0,1, the Raman gain was compared. This multimode amplification is mainly used in space-division multiplexing (Antonelli, Mecozzi \& Shtaif 2013; Namiki \& Emori, 2001). We can make any fiber into a Raman amplifier by suitable selection of pumping wavelength (Anwar \& Aly, 2010). This paper investigates two important views in RA. Section 2 investigates about RA performance in various Refractive Index Profiles and Section 3 investigates about the performance of RA with EDFA hybrid configuration.

\section{INVESTIGATIONS OF REFRACTIVE INDEX PROFILE (RIP)}

Raman amplifiers are used not only for amplification but also for dispersion compensation. In Dispersion compensation, the refractive index profile of fiber plays a major role.

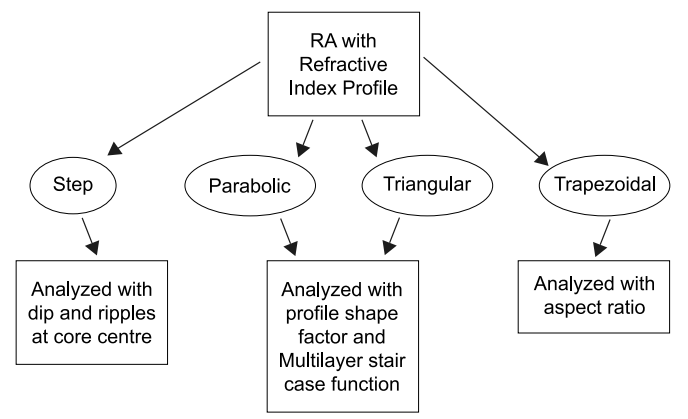

Figure 1. Study of Raman Amplifier based on Refractive Index Profile.

In the design of RA, the operating wavelength range is adjusted by correctly selecting suitable RIP structure and inner core radius. In this paper, RIP based performance of RA is studied as per Figure 1 Among step, parabolic and triangular refractive index profiles, a parabolic profile provides good effective Raman gain results at 20Gbps. The paper (Chan \& Premaratne 2007) also reports the change in dispersion co-efficient and gain as the function of RIP. For obtaining better flattening of Raman gain, the core gap radius of a fiber is varied with step index profile. Additional to this, large negative dispersion coefficient is achieved by varying core gap radius (Bandyopadhyay \& Sarkar 2013) From this, dispersion compensation is achieved at C and S bands. A research (Pramanik, Das, \& Sarkar, 2010) 
found that Trapezoidal index profile also affects the gain performance of RA. The aspect ratio $\mathrm{S}$ is modified, and same phase matching is achieved for various values of core radius. During the design of RA, step index profile at both inner and outer cores was considered in another research (Pramanik \& Sarkar, 2014). This work says that the occurrence of axial dip at core center is unavoidable. Up to $0.25 \%$ dip depth and $25 \%$ dip width, RA is performing as perfect as RA without any dip or profile imperfections. But above this limit of dip depth and dip width, the performance becomes poor. This paper concluded that step index profile in core is most excellent for better performance of RA.

From the investigations of these research works, the Raman gain of various profiles are observed at $1550 \mathrm{~nm}$ wavelength window for fundamental mode $\operatorname{LP}(0,1)$ and tabulated in Table 1.

Table 1. Raman Gain values at 1550nm Window.

\begin{tabular}{|c|c|}
\hline Type of profile & Raman gain $(\mathbf{m} / \mathbf{W})$ \\
\hline Parabolic index & $0.7 \times 10-13$ \\
\hline Triangular index & $0.62 \times 10-13$ \\
\hline Step index & $0.76 \times 10-13$ \\
\hline Trapezoidal index & $1.04 \times 10-13$ \\
\hline
\end{tabular}

From this Table 1, trapezoidal index profile gives the better Raman gain. But even with dip at core centre step index profile gives the better performance (Pramanik et al., 2014).

\section{INVESTIGATIONS ON HYBRID COMBINATION OF EDFA \& RAMAN AMPLIFIERS}

Many research works have used benefits from both the fiber amplifiers RA and EDFA. When RA is combined with EDFA, cross talk becomes very less even for channel spacing of $0.4 \mathrm{~nm}$ and $0.2 \mathrm{~nm}$. This hybrid RA and EDFA performance is inspected for 16X10Gbps DWDM system (Singh \& Kaler, 2015). And this paper concluded that hybrid RA and EDFA is better than other hybrid optical amplifiers. Better power utilization also possible with this hybrid RA and EDFA. It is investigated in the research (Lee, Chang, Han, Kim, Chung \& Lee, 2004) that recycling the residual Raman pump to make pumping on EDFA. Choosing proper pump wavelength is the only thing to be considered. This gives the possibility for the design of broadband amplifiers with high gain. 


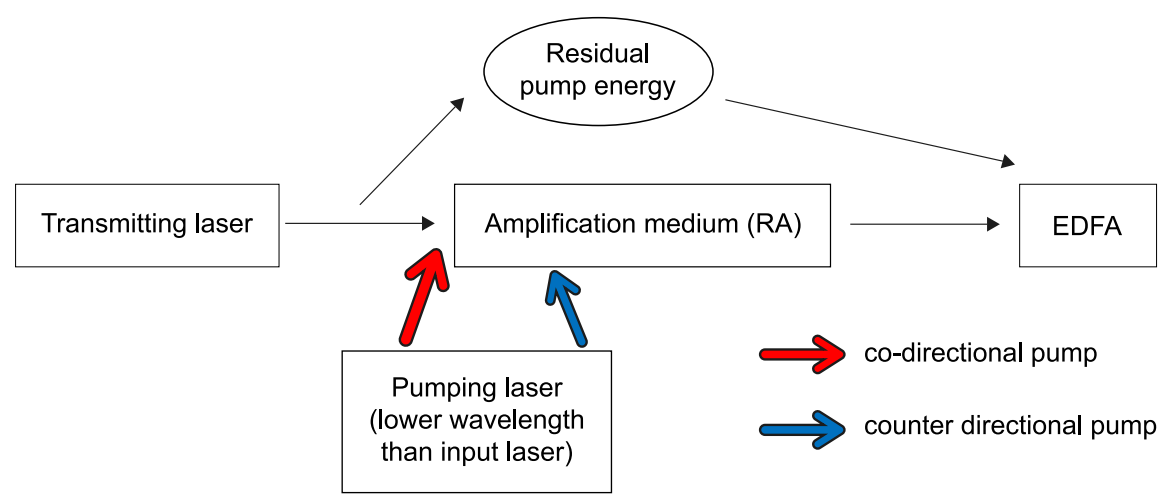

Figure 2. RA-EDFA hybrid configuration with single pump.

As shown in Figure 2, the pumping may be given either in forward direction (co-direction) or in backward direction (counter-direction).

In hybrid optical amplifiers, the site of the Erbium doped fiber severely affects the performance of the dispersion compensation of Raman/EDF and this is discussed in a research (Ali, Abdullah, Jamaludin, Al-Mansoori, Al-Mashhadani \& Abass, 2013). EDF may be placed before RA or after RA. In this work, these are considered as two cases. A 10m EDF and $7 \mathrm{Km}$ Dispersion Compensating Fiber (DCF) is considered. Here, DGF is acting as Raman Amplification medium. In first case, EDF is placed before RA. And in second case EDF is placed after RA. In this research, fiber has the following specifications. They are $0.55 \mathrm{~dB} / \mathrm{Km}$ of attenuation coefficient, $98 \mathrm{ps} / \mathrm{nm} . \mathrm{km}$ of dispersion co efficient, and 15.3 $\mu \mathrm{m} 2$ of effective area. And EDF has the following specifications. Erbium concentration of $440 \mathrm{ppm}, 2.2 \mu \mathrm{m}$ core radius, and effective area of $15.2 \mu \mathrm{m}^{2}$. If we place the RA firstly and EDFA secondly, then we obtain large input signal gain, noise figure and gain variation. RAMAN-SOA and RAMAN -EDFA are analyzed in the research of Upma (2015). This research proposed the 8 channel transmitter with constant attenuation value $0.2 \mathrm{db} / \mathrm{Km}$. And data speed of $10 \mathrm{Gbps}$ is considered. Under such case, Raman-EDFA gives highest $\mathrm{Q}$ factor of 19.92db, RAMAN-SOA provides highest eye opening, Raman -EDFA provides smallest jitter 0.0243. so RAMAN -EDFA is a hopeful alternative to all other hybrid amplifiers. With the help of mono pump source, dispersion compensating Raman/EDFA hybrid amplifier is achieved. Also overall power conversion efficiency is increased. Here mono pump source having two lasers is used for Dispersion Compensating Fiber (DCF) which has Raman Amplification. The remaining power from this DCF is again recycled 
and utilized by EDFA. The laser pumps are operating at $1455 \mathrm{~nm}$ and $1465 \mathrm{~nm}$. A total pump power of $500 \mathrm{~mW}$ is launched into a $12.6 \mathrm{Km}$ DCF. Effective power utilization and larger amplifier efficiency is obtained in the research (Lee, Chang, Han, Chung, Kim \& Lee, 2005).

Like Erbium, Ytterbium can also be used as fiber amplifier. By Yb-Raman combined non linear amplifier, an improved power of $1.5 \mathrm{~kW}$ was obtained in the wavelength of $1100 \mathrm{~nm}$ to $1200 \mathrm{~nm}$. In this work (Zhan, Tao, Zhou, Wang, \& Xu, 2014), the amplifier is seeded by 1070 and 1120nm signal lasers simultaneously.

For 16 channel system (Lee, Oh, Lee, Lee, \& Hwang, 2004), Q factor \& BER for RamanEDFA \& EDFA-RAMAN-EDFA are same for short distance. For longer distance, EDFARAMAN-EDFA has largest $Q$ value among all. For 32 channel system, RAMAN-EDFA provides good output power; BER \& Q factor. In the hybrid combination of RamanEDFA, EDFA may be used in parallel configuration and residual pumping is done. In order to minimize the cost, mono pump wavelength is used in Raman amplifier. Raman amplifier in the role of DCF has two benefits. First one is, obtaining low loss and dispersion compensation at the same time. Second is, amplification is done at wider band in optical wavelength window by changing the pump wavelength and it was discussed in a research (Singh \& Kaler, 2015). In order to maximize capacity for amplification scheme and transmission distance, a variety of combinations of three 16QAM based coded modulation schemes with spectral efficiencies 4.86/5.4 for C+L EDFA experiment and 5.45/6.14 [bits/ $\mathrm{Hz}]$ are used in this paper (Cai et al., 2015).

A different research is done in this work (Mahran, 2015). Here, the bending loss in EDFA makes the gain of hybrid amplifier to increase up to 7db more than normal EDFA/Raman. OSNR calculations also show a better performance. In this paper bending radius is chosen as $4 \mathrm{~mm}$, EDFA is taken with length $10 \mathrm{~m}$ where, forward pump power in the range of 100 $500 \mathrm{~mW}$, Raman amplifier length is chosen between 12 to55Km, where backward pump power in the range of $80-200 \mathrm{mw}$ and input signal power is $-20 \mathrm{dBm}$. Even for reduced channel spacing, this hybrid RA-EDFA provides better performance. This research (Singh \& Kaler, 2012) investigated various combination of hybrid amplifiers. 100 channels were 
used at channel spacing of $6.25 \mathrm{GHz}$. Quality factors, acceptable bit rate, Bit Error Rate (BER) were calculated for all the configurations.

Pump power of RA is unused in Dispersion compensating modules (DCM). This wastage of pump power is avoided and utilized by using FBG at one side of DCM. This DCM is designed for short or medium distance mostly from $50 \mathrm{Km}$ to $100 \mathrm{Km}$ (Nicholson, 2003). Both the benefits of dispersion compensation and effective power utilization were obtained from this research.

\section{SUMMARY}

This paper assesses about various perspectives of Raman Amplifiers. The effort towards mitigating dispersion, improving gain bandwidth, effective power utilization, obtained low noise performance, dependency of Refractive index profile using RA and corresponding researches were described in this paper. When hybrid configuration of RA-EDFA is considered, the innovative research papers of bending loss in EDFA for dispersion compensation and locating EDFA for low noise were also depicted in this paper. 


\section{REFERENCES}

Ali, M. H., Abdullah, F., Jamaludin, M. Z., Al-Mansoori, M. H., Al-Mashhadani, T. F., \& Abass, A. K. (2013). Effect of EDF position on the performance of hybrid dispersion-compensating Raman/EDF amplifier. In IEEE 4th International Conference on Photonics (ICP) (pp. 187-189). IEEE. https://doi.org/10.1109/ICP.2013.6687109

Antonelli, G., Mecozzi, A., \& Shtaif, M. (2013). Raman amplification in multimode fibers with random mode coupling. Optics letters, 38(8), 1188-1190. https://doi. org/10.1364/OL.38.001188

Anwar, N. M., \& Aly, M. H. (2010). Backward Pumped Distributed Fiber Raman Amplifiers. 27th National Radio Science Conference. https://www.researchgate.net/ publication/259181526_Backward_Pumped_Distributed_Fiber_Raman_ Amplifiers

Bandyopadhyay, P. K., \& Sarkar, S. (2013). Effect of variation of core gap radius on the performance of dual concentric core Raman fiber amplifier. Optics Communications, 300, 27-32. https://doi.org/10.1016/j.optcom.2013.02.029

Bromage, J. (2004). Raman amplification for fiber communications systems. Fournal of Lightwave Technology, 22(1), 79-93. https://doi.org/10.1109/JLT.2003.822828

Cai, J. X., Sun, Y., Zhang, H., Batshon, H. G., Mazurczyk, M. V., Sinkin, O. V., ... \& Pilipetskii, A. (2015). $49.3 \mathrm{~Tb} / \mathrm{s}$ transmission over $9100 \mathrm{~km}$ using C+ L EDFA and $54 \mathrm{~Tb} / \mathrm{s}$ transmission over $9150 \mathrm{~km}$ using hybrid-Raman EDFA. Journal of Lightwave Technology, 33(13), 2724-2734. https://doi.org/10.1109/JLT.2015.2409846

Chan, A. C. O., \& Premaratne, M. (2007). Dispersion-compensating fiber Raman amplifiers with step, parabolic, and triangular refractive index profiles. Fournal of lightwave technology, 25(5), 1190-1197. https://doi.org/10.1109/JLT.2007.893033

Dhir, E. J., \& Gupta, E. V. (2013). Improvement of Gain with Figure of Merit in Discrete Raman Amplifier. International Fournal of Computer Science and Communication Engineering, (IFCSCE), 22-24. 
Dhir, J., \& Gupta, V. (2014). Improvement of Raman Gain with Different Parameters in Discrete Raman Amplifier. International Journal of Engineering Research and Applications (IJERA). National Conference on Advances in Engineering and Technology (pp. 16-19). http:// www.ijera.com/special_issue/AET_Mar_2014/ECE/Version\%20\%201/D1619. pdf

Elndash, A., Mohammed, N. A., \& Rashed, A. N. Z. (2010). Comparison performance evolution of different transmission techniques with bi-directional distributed Raman gain amplification technique in high capacity optical networks. International Journal of Physical Sciences, 5(5), 484-495. https://www.semanticscholar.org/paper/ Comparison-performance-evolution-of-different-with-Elndash-Mohammed/7c085 d6ce3d37b342167a0bb8e624eceea036646

Ferreira, G. G., Gani, S. P. N., Pontes, M.J., \& Segatto, M. E. V. (2011). Optimization of distributed Raman amplifiers using a hybrid genetic algorithm with geometric compensation technique. IEEE Photonics Fournal, 3(3), 390-399. https://doi. org/10.1109/JPHOT.2011.2140366

Fugihara, M. G., \& Pinto, A. N. (2008). Low-cost Raman amplifier for CWDM systems. Microwave and Optical Technology Letters, 50(2), 297-301. https://doi.org/10.1002/ mop. 23075

Islam, M. N. (2002). Raman amplifiers for telecommunications. IEEE fournal of selected topics in Quantum Electronics, 8(3), 548-559. https://doi.org/10.1109/JSTQE.2002.1016358

Lee, H. H., Oh, J. M., Lee, D., Lee, G. W., \& Hwang, S. T. (2004). Demonstration of $16 \times 10 \mathrm{~Gb} / \mathrm{s}$ WDM transmission over $4 \times 40 \mathrm{~km}$ of SMF using linear optical amplifiers combined with Raman-pumped dispersion compensation fibers under dynamic add/drop situations. In Optical Fiber Communication Conference, Technical Digest (CD) (Optical Society of America, 2004), paper WG2. https://www.osapublishing.org/ abstract.cfm? uri $=\mathrm{OFG}-2004-\mathrm{WG} 2$ 
Lee, J. H., Chang, Y. M., Han, Y. G., Ghung, H., Kim, S. H., \& Lee, S. B. (2005). A detailed experimental study on single-pump Raman/EDFA hybrid amplifiers: static, dynamic, and system performance comparison. Fournal of light wave technology, 23(11), 3484. https://doi.org/10.1109/JLT.2005.857773

Lee, J. H., Chang, Y. M., Han, Y. G., Kim, S. H., Ghung, H., \& Lee, S. B. (2004). Dispersion-compensating Raman/EDFA hybrid amplifier recycling residual Raman pump for efficiency enhancement. IEEE Photonics Technology Letters, 17(1), 43-45. https://doi.org/10.1109/LPT.2004.837264

Mahran, O. (2015). Performance study of macro-bending EDFA/Raman hybrid optical fiber amplifiers. Optics Communications, 353, 158-164. https://doi.org/10.1016/j. optcom.2015.05.030

Namiki, S., \& Emori, Y. (2001). Ultrabroad-band Raman amplifiers pumped and gainequalized by wavelength-division-multiplexed high-power laser diodes. IEEE fournal of Selected Topics in Quantum Electronics, 7(1), 3-16. https://doi.org/10.1109/2944.924003

Neto, B., Teixeira, A. J., Wada, N., \& André, P. S. (2007). Efficient use of hybrid genetic algorithms in the gain optimization of distributed Raman amplifiers. Optics express, 15(26), 17520-17528. https://doi.org/10.1364/OE.15.017520

Nicholson, J. W. (2003). Dispersion compensating Raman amplifiers with pump reflectors for increased efficiency. Fournal of lightwave technology, 21(8), 1758. https://doi. org/10.1109/JLT.2003.815495

Polley, A., \& Ralph, S. E. (2007). Raman amplification in multimode fiber. IEEE Photonics Technology Letters, 19(4), 218-220. https://doi.org/10.1109/LPT.2006.890752

Pramanik, S., \& Sarkar, S. (2014). Effect of dip in refractive index profile on fiber Raman gain amplifier performance. Optics Communications, 329, 145-150. https:// doi.org/10.1016/j.optcom.2014.05.016

Pramanik, S., Das, G., \& Sarkar, S. N. (2010). Comparative study of the influence of the aspect ratio of trapezoidal index profiles on the performance of a fiber Raman amplifier. Optical Engineering, 49(5), 055001. https://doi.org/10.1117/1.3421554 
Singh, S., \& Kaler, R. S. (2012). Investigation of hybrid optical amplifiers for dense wavelength division multiplexed system with reduced spacings at higher bit rates. Fiber and Integrated Optics, 31(3), 208-220. https://doi.org/10.1080/01468030.2012 .666818

Singh, S., \& Kaler, R. S. (2015). Performance optimization of EDFA-Raman hybrid optical amplifier using genetic algorithm. Optics \& Laser Technology, 68, 89-95. doi: https://doi.org/10.1016/j.optlastec.2014.10.011

Singh, S., \& Kaler, R. S. (2015). Review on recent developments in hybrid optical amplifier for dense wavelength division multiplexed system. Optical Engineering, 54(10), 100901. https://doi.org/10.1117/1.OE.54.10.100901

Upma, P. S. (2015). Performance Comparison Of Optical Amplifiers And their Hybrid Configurations In 8x10 Gbps WDM Based Optical Network. International fournal of Engineering Research and General Science, 3(3), 154-158. http://pnrsolution.org/ Datacenter/Vol3/Issue3/233.pdf

Zaki Rashed, A. N. (2011). New trends of forward fiber Raman amplification for Dense Wavelength Division Multiplexing (DWDM) photonic communication networks. International Fournal on Technical and Physical Problems of Engineering, 3, 30-39. http:// www.iotpe.com/IJTPE/IJTPE-2011/IJTPE-Issue7-Vol3-No2-Jun2011/6-IJTPEIssue7-Vol3-No2-Jun2011-pp30-39.pdf

Zhang, H., Tao, R., Zhou, P., Wang, X., \& Xu, X. (2014). 1.5-kW Yb-Raman combined nonlinear fiber amplifier at $1120 \mathrm{~nm}$. IEEE Photonics Technology Letters, 27(6), 628-630. https://doi.org/10.1109/LPT.2014.2386973 
